\title{
Defect Character at Grain Boundary Facet Junctions: A Combined HRSTEM and Atomistic Modeling Study of a $\Sigma=5$ Grain Boundary in Fe
}

\author{
D.L. Medlin ${ }^{1}$, K. Hattar ${ }^{2}$, J. Zimmerman ${ }^{1}$, F. Abdeljawad ${ }^{2}$, and S.M. Foiles ${ }^{2}$ \\ ${ }^{1}$ Sandia National Laboratories, Livermore, California 94551, USA \\ ${ }^{2}$ Sandia National Laboratories, Albuquerque, New Mexico 87185, USA
}

Electron microscopy plays an important role in motivating and testing our theoretical understanding of grain boundary structure and behavior across the atomistic and continuum length scales. One of the key foundational challenges in grain boundary science is to establish meaningful links between atomic-scale interfacial configurations, which can often be described in terms of sets of characteristic structural units [1], and more macro-scale descriptors of the interfacial geometry, such as grain misorientation and boundary inclination. A useful approach for establishing such linkages is to identify and to characterize the sets of interfacial line defects that accommodate departures from low-energy singular reference configurations [2].

In this presentation, we consider such approaches in the context of atomic resolution experimental observations and theoretical calculations of $\Sigma=5<001>$ tilt boundaries in BCC iron. We focus in particular on the analysis of facet junctions and grain boundary dislocations. Figure 1a shows a high angle annular dark field (HAADF) scanning transmission electron micrograph (STEM) of such a boundary observed in an annealed pulsed-laser-deposited thin film of Fe. The boundary has formed nanoscale facets lying on $\{310\}$ and $\{210\}$ planes, which correspond to the two types of symmetric inclination that are geometrically possible for $\Sigma=5<001>$ tilt boundaries. We have conducted atomistic simulations for these inclinations using several interatomic potentials and density functional theory and have compared these calculations with the observed structures. Examples showing structures calculated using the potential of Mendelev et al. [3] are shown in Figures 1b and 1c. Our calculations predict these inclinations to be cusps in the energy versus inclination landscape, which is consistent with our observation of faceting on these planes.

Measurements of the boundary misorientation show a small deviation $\left(2.4^{\circ}\right)$ from the exact $\Sigma=5$ alignment. By analyzing the boundary dislocation content (Figure 2) we have shown that this angular deviation is accommodated by an array of $(1 / 5)[3,1,0]$ and $(1 / 5)[1,2,0]$ dislocations distributed at the facet junctions in a manner consistent with the observed average inclination. These atomistic observations help in challenging our thinking about the interplay between dislocation and boundary faceting energetics. To illustrate, we will discuss how this analysis is being used to motivate and to refine a continuum phase-field approach for modeling the effects of junction dislocation character on the energetics and kinetics of grain boundary facet coarsening.

[1] A.P. Sutton and V. Vitek, Phil. Trans. Royal Society A 309 (1983) 1-68.

[2] R.C. Pond, in Dislocations in Solids (Ch. 38) (Ed. F.R.N. Nabarro, Elsevier, 1989).

[3] M.I. Mendelev, et al., Philosophical Magazine 83 (35) (2003) 3977-3994.

[4] C.L. Kelchner, S.J.Plimpton, J.C. Hamilton, Phys. Rev. B 58 (1998) 11085.

[5] Sandia National Laboratories is a multi-program laboratory managed and operated by Sandia Corporation, a wholly owned subsidiary of Lockheed Martin Corporation, for the U.S. Department of Energy's National Nuclear Security Administration under contract DE-AC04-94AL85000. 

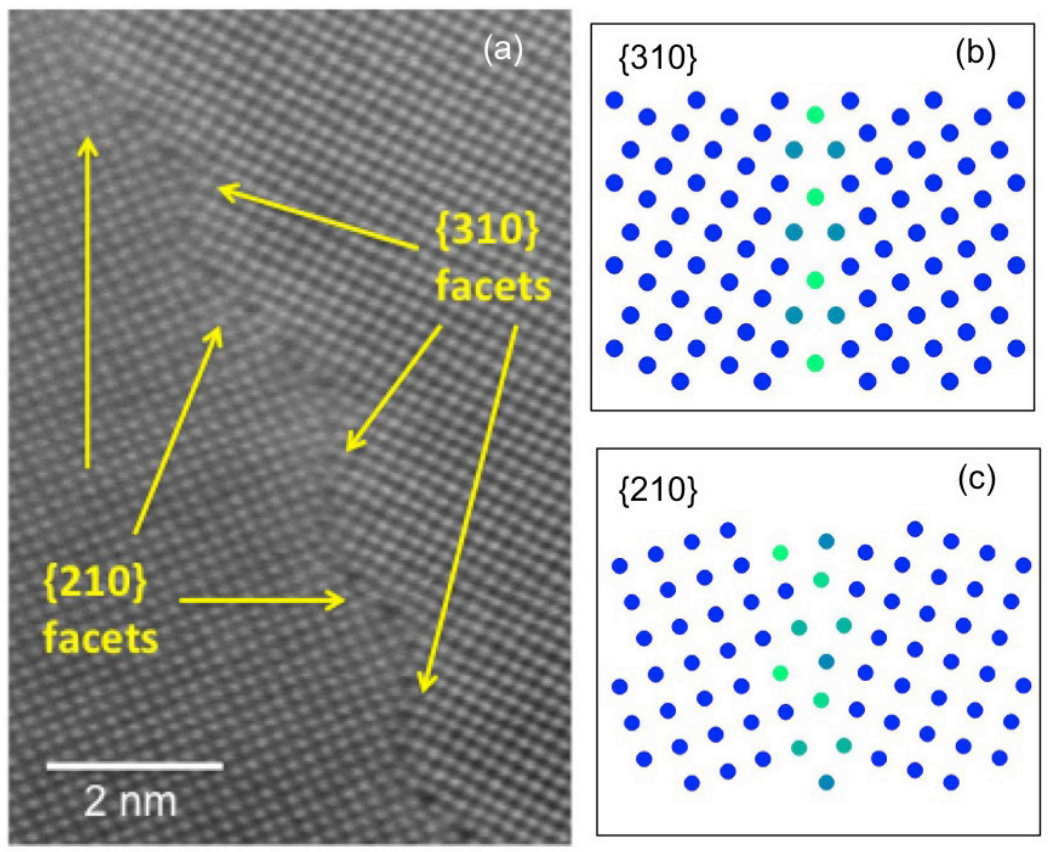

Figure 1. (a) HAADF-STEM image of a $\Sigma=5$ [001] boundary in Fe. The boundary has formed nanoscale facets on $\{310\}$ and $\{210\}$ type planes. Figures (b) and (c) show atomic structures for the ideal $\Sigma=5\{310\}$ and $\{210\}$ boundaries as calculated using the Mendelev potential [3] and shaded by the centro-symmetry parameter [4].

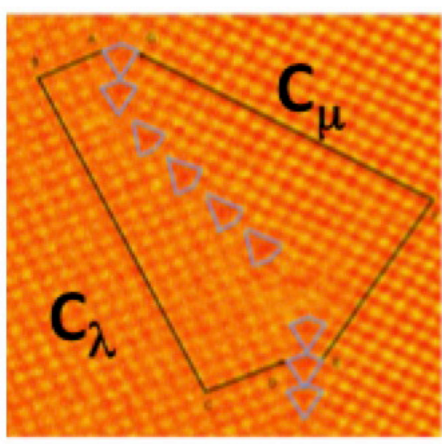

(a)

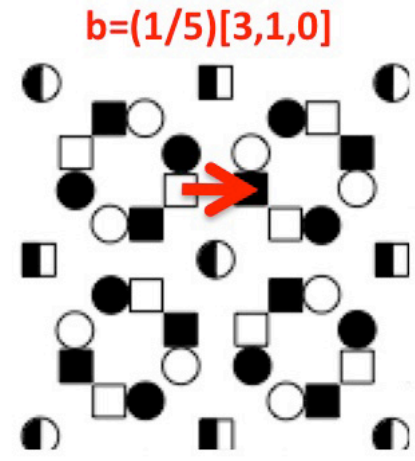

(b)

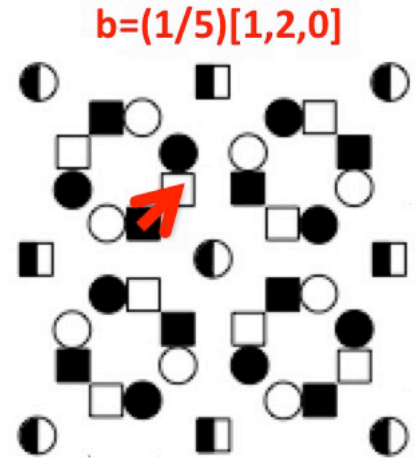

(c)

Figure 2. (a) The dislocation content at the facet junctions was determined by circuit mapping. Here the circuit is separated into two paths, $C_{\lambda}$ and $C_{\mu}$. The Burgers vector is calculated from $\mathbf{b}=-\left(C_{\lambda}+\mathbf{P} C_{\mu}\right)$, where $\mathbf{P}$ is a matrix transformation re-expressing path $C_{\mu}$ into the crystal coordinates of crystal $\lambda$. The analysis identifies grain boundary dislocations that are located at the facet junctions and possess Burgers vectors of type $(1 / 5)[3,1,0](b)$ and $(1 / 5)[1,2,0]$ (c). These Burgers vectors are plotted here on the $\Sigma=5$ dichromatic pattern, which shows the superposition of the two BCC crystal lattices. 